\title{
ESTRÉS, ANSIEDAD Y DEPRESIÓN EN MAGISTRADOS DE LIMA, PERÚ
}

\author{
Alberto Perales ${ }^{1,2, a}$, Héctor Chue ${ }^{3, b}$, Alberto Padilla ${ }^{4, c}$, Lorenzo Barahona ${ }^{5, d}$
}

\begin{abstract}
RESUMEN
Objetivos. Determinar los niveles de estrés general y laboral, depresión y ansiedad en magistrados (jueces y fiscales) del Distrito Judicial de Lima, Perú. Materiales y métodos. Estudio transversal y descriptivo. De una población de 1137 magistrados del Poder Judicial y del Ministerio Público del Distrito Jurisdiccional de Lima se tomó una muestra aleatoria estratificada donde cada institución constituyó un estrato. La muestra estuvo conformada por 287 magistrados: 138 fiscales y 149 jueces. Se aplicó un cuestionario compuesto por cuatro instrumentos para determinar los niveles de estrés general y laboral, ansiedad y depresión, previo consentimiento informado. Resultados. El estrés general estuvo presente en el $18,5 \%$ de los magistrados, el estrés laboral en $33,7 \%$, ansiedad en 12,5 \% y depresión en $15,0 \%$. Estas tres condiciones estuvieron presentes en el $6,6 \%$ de los magistrados, y al menos una estuvo presente en el 25,9\%. El estrés general estaba asociado con la depresión (OR: 4,9; IC95 \% 1,6-15,1) y ansiedad (OR: 8,5; IC95 \% 2,5-28,6) en el modelo de regresión logística. Conclusiones. Un porcentaje significativo de magistrados del Distrito Judicial de Lima presentan altos niveles de estrés, ansiedad y depresión; un cuarto de los encuestados presentan, al menos, una de estas condiciones. Se recomienda que los programas de intervención y prevención se dirijan al manejo del estrés, ansiedad y depresión en conjunto, dada su alta asociación.
\end{abstract}

Palabras clave: Sistema de justicia; Poder judicial; Estrés laboral; Depresión; Ansiedad; Salud mental (fuente: DeCS BIREME).

\section{STRESS, ANXIETY AND DEPRESSION IN MAGISTRATES FROM LIMA, PERU}

\begin{abstract}
Objective. To measure the general and work related stress, depression and anxiety in Magistrates (Judges and District Attorneys) of Lima Judicial District. Material and methods. We carried out a transversal and descriptive study. A population of 1137 magistrates from the Lima Jurisdictional District of the Judicial System, was randomly sampled by a strata representing every agency of the Judicial System. The final sample included 287 magistrates: 138 District Attorneys and 149 Judges. After informed consent, a questionnaire composed by four previously validated instruments was applied to measure general and work related stress, anxiety and depression. Results. General stress was present in $18.5 \%$ of the participants; work related stress $33.7 \%$; anxiety $12.5 \%$, and depression $15 \%$. All three conditions were present in $6.6 \%$ of the participants and at least one in $25.9 \%$. General stress was associated to depression (OR: $4.9 ;$ IC95 \% 1.6-15.1) and anxiety (OR: 8.5; IC95 \% 2.5-28.6) by logistic regression. Conclusion. Lima Judicial District's Magistrates present a triad composed by high levels of labor stress, anxiety and depression. A quarter of the participants present at least one of those conditions. In that perspective, it is recommended that intervention and preventive programs address stress, anxiety and depression simultaneously, given their strong association.
\end{abstract}

Key words. Justice administration system; Judicial power; Burnout, professional; Depression; Anxiety; Mental health (source: MeSH NLM).

\section{INTRODUCCIÓN}

En el campo laboral, es conocido que el estrés produce daños en la salud con su consecuente impacto negativo en la productividad de los trabajadores ${ }^{(1)}$. En el área de la magistratura peruana, desde diciembre de 2005 hasta agosto de 2008, Perales A. y Chue H., realizaron un estudio preliminar en una muestra conformada por 109 magistrados, participantes del proceso de evaluación y ratificación del Consejo Nacional de la Magistratura

\footnotetext{
Instituto de Ética en Salud, Facultad de Medicina, Universidad Nacional Mayor de San Marcos. Lima, Perú.

Academia Nacional de Medicina. Lima, Perú.

PSICOSALUD SAC. Lima, Perú.

Facultad de Salud Pública y Administración, Universidad Peruana Cayetano Heredia. Lima, Perú.

5 Facultad de Medicina, Universidad Nacional Mayor de San Marcos. Lima, Perú.

a Médico Psiquiatra, Doctor en Medicina, Diplomado en Ética y Deontología en Salud; ' ${ }^{b}$ Médico Psiquiatra; ${ }^{\circ}$ Estadístico, postgrado en demografía y salud pública; ${ }^{\mathrm{d}}$ Médico Psiquiatra, Magister en Medicina
}

Recibido: 12-04-11 Aprobado: 07-12-11 
(CNM); de ellos, el $62 \%$ informó experiencias subjetivas de elevado estrés y, en el aspecto físico, el 61 \% informó padecer de diversas enfermedades médicas (hipertensión arterial, trastornos digestivos, sobrepeso y otras). Así mismo, la prevalencia de vida para trastornos de ansiedad fue del 27 y $35 \%$ para trastornos depresivos ${ }^{(2)}$. Este escenario tan preocupante motivó al CNM, en concordancia con el Poder Judicial (PJ) y el Ministerio Público (MP), a evaluar el problema con la finalidad de determinar su importancia y proponer, de ser necesario, un programa de intervención preventivo y correctivo que proteja la salud integral de los magistrados para que cumplan con sus funciones en las mejores condiciones.

Sin embargo, la investigación científica en magistrados es prácticamente inexistente en el país. En tanto, en el ámbito internacional, Brerner ${ }^{(3)}$, realizó un estudio en magistrados de Estados Unidos, en el que evaluó el impacto de un programa que identifica estrés ocupacional, tensión personal y fuentes de afrontamiento; encontró que el estrés ocupacional se manifiesta como tensión física y mental, sintomatología que podría reducirse de ofrecer al juez una fuente de soporte social, tal como un mentor o guía. Luego de un periodo de cinco meses, en la que se designaron jueces especialmente capacitados para dar soporte social a los magistrados recientemente nombrados, los resultados señalaron, inicialmente, un bajo puntaje de estrés y de tensión, y elevado puntaje en habilidades de afronte. Concluyeron así, que el soporte social dado por los mentores debería ser considerado en la formación tradicional de los jueces.

Novaes y Sacramento realizaron en Brasil un estudio sobre estrés ocupacional, fuentes de estrés y estrategias de afrontamiento en jueces laborales. El $71 \%$ de los jueces presentó síntomas significativos de estrés, con afectación de su calidad de vida, particularmente en las áreas afectiva y de salud general. La mayor fuente de estrés es el número excesivo de procesos a juzgar; la estrategia de afronte más usada fue conversar con el o la cónyuge, o con una persona a quien estimara de forma particular. Los síntomas de estrés más frecuentes fueron la sensación de agotamiento, cansancio y tensión muscular; pero solo una minoría mencionó el uso de terapia, el deporte o técnicas de relajación. Se concluyó que los jueces laborales tenían una percepción de su profesión similar a la de los trabajadores de minas o pilotos de avión. Por ello, los autores plantean una especial atención preventiva y de aprendizaje de estrategias de afrontamiento al estrés ${ }^{(4)}$.

Estos resultados son compartidos por Pereira da Silva, que identificó factores que desencadenan el estrés en 930 magistrados del Tribunal de Justicia del Estado de Minas Gerais, Brasil. Sus resultados señalan que la percepción de estrés estuvo influenciada por los cambios sociales, el avance tecnológico y la globalización, que contribuyen y favorecen la acumulación de trabajo judicial ${ }^{(5)}$.

Hunter, al evaluar el estrés judicial y establecer estresores para asociarlos con el desempeño laboral, encuentra una relación significativa entre el incremento de los síntomas de Burnout (agotamiento extremo físico y mental) y el incremento de los síntomas del estrés ${ }^{(6)}$.

Por otro lado, el avance tecnológico se ha convertido en un estresor para los magistrados. Salanova menciona que: Internet, la telefonía móvil, el tele-trabajo y la sociedad de la información, son cada vez más familiares, constituyéndose en estresores tecnológicos que afectan tanto la vida privada como pública; añadiendo un riesgo más a la carga mental de los magistrados como sobrecarga informativa ${ }^{(7)}$.

La Psiquiatría actual postula una relación entre estrés y depresión ${ }^{\left({ }^{8}\right)}$ y entre estrés y ansiedad ${ }^{(9)}$. Por todo ello, y dada la falta de información sobre este tema en el Perú, el objetivo principal del presente trabajo es determinar los niveles de estrés general y laboral, de ansiedad y de depresión en magistrados (jueces y fiscales) de una muestra representativa del Distrito Judicial de Lima.

\section{MATERIALES Y MÉTODOS}

Se realizó un estudio observacional y descriptivo, cuyo universo estuvo constituido por los magistrados del Distrito Judicial de Lima, que se encontraban en actividad en el momento de la investigación (abril, 2009), considerando entre ellos a los del magistrados del Ministerio Público y del Poder Judicial. La población total comprendió 1137 magistrados, siendo 635 del Ministerio Público y 502 del Poder Judicial.

El tamaño de la muestra fue determinado considerando un nivel de confianza del $95 \%$, un margen de error relativo del $10 \%$, con una proporción de estrés general del $50 \%$, con una corrección para poblaciones finitas. La muestra estuvo constituida por 287 magistrados (138 del Ministerio Público y 149 del Poder Judicial), los cuales constituyen el $25 \%$ del total de magistrados del ámbito mencionado. Se empleó un muestreo estratificado (el Ministerio Público y el Poder Judicial constituyeron un estrato cada uno), sistemático y aleatorio, independiente en cada estrato, con una asignación proporcional al número de magistrados de cada institución. El marco de muestreo para la selección de los magistrados estuvo constituido por la relación de magistrados de cada institución, proporcionadas por el CNM. 
Se aseguró el anonimato de los informantes, empleando un código individual que permitió relacionar los resultados obtenidos. Asimismo, previo al estudio, se solicitó el consentimiento informado a cada participante. El estudio contó con la aprobación del CNM.

\section{INSTRUMENTOS}

Se empleó un cuestionario autoadministrado y estructurado de cinco partes, cada una permitía la evaluación de un aspecto específico del estudio: datos generales, estrés general, depresión, ansiedad y estrés laboral en magistrados. Cada sección fue elaborada según las siguientes consideraciones:

Breve cuestionario sobre el estrés general ${ }^{(10)}$. Consta de trece ítems que tienen un puntaje de 0 a 4 que discriminan el nivel de estrés; de acuerdo con sus instrucciones, el punto de corte para identificar a personas que no manejan adecuadamente el estrés es 15 .

Escala de autoevaluación de la depresión de Zung (11). Consta de veinte ítems y discrimina síntomas de depresión (punto de corte: 50). Validada en el Perú por Novara et al (12).

Escala de automedición de la ansiedad de Zung ${ }^{(13,14)}$. Consta de veinte ítems y discrimina síntomas de ansiedad (punto de corte: 50 ), se usó la versión validada al español.

Cuestionario de factores de estrés laboral para magistrados. Cuestionario construido ad hoc para el presente estudio por Perales A, Chue $\mathrm{H}$, Padilla A y Barahona L, en el 2008. Esta sección fue construida en base a temas identificados en una sesión dirigida en la que participaron 35 magistrados, como parte de las actividades preparatorias de la investigación y prueba de instrumentos. En dicha sesión se identificaron quince problemas que se transformaron en preguntas. Cada una de ellas consta, a su vez, de cuatro opciones de respuesta ( $1=$ raramente a $4=$ siempre).

Mediante la aplicación del análisis de componentes principales, se determinaron cuatro componentes que explicaban el $61,9 \%$ de la varianza total de los datos. Tales dimensiones fueron:

a) Los aspectos relacionados con el ambiente laboral (organización, carga procesal, personal y apoyo logístico, infraestructura institucional y relaciones interpersonales).

b) La presión social a la que están sometidos los magistrados, por parte de la opinión pública y los abogados litigantes. c) Los factores relacionados con la superación profesional y la estabilidad en el cargo, destacando la fuerte presión que experimentan los magistrados por el proceso de evaluación y ratificación a cargo del Consejo Nacional de la Magistratura; el esfuerzo que les demanda los estudios (posgrado y otros) necesarios para avanzar académica y profesionalmente, así como para adquirir nuevos conocimientos para resolver la complejidad de los casos.

d) El trato que reciben de parte de sus superiores.

En una sesión posterior el cuestionario fue aplicado a otro grupo de magistrados con cuyas respuestas se construyó una base de datos y se hizo el análisis de consistencia interna empleando el coeficiente alfa de Cronbach, que fue de 0,863 .

La escala permitía una calificación de 15 a 60 puntos; al realizar la distribución de los valores obtenidos en el piloto, decidimos colocar como punto de corte 33 puntos, pues sobre dicho valor se encontraba un tercio de la población participante; a este grupo lo calificamos como posibles portadores de estrés laboral.

La evaluación de los magistrados se realizó en dos momentos. En el primero, un grupo de enfermeras midió el peso, estatura y presión arterial de cada magistrado con instrumentos calibrados de uso médico. En segundo lugar, los magistrados procedieron a llenar los cuestionarios. Luego se realizó el análisis de la información.

\section{PROCESAMIENTO DE LOS DATOS}

Para la determinación de los niveles de estrés, de ansiedad y de depresión se sumaron los puntajes obtenidos en cada una de las preguntas de las secciones correspondientes de la encuesta para luego, siguiendo las recomendaciones de los autores de los respectivos instrumentos, clasificarlos en dos niveles, normal y alto, en cada uno de ellos.

Se hicieron estimaciones puntuales y de intervalos al $95 \%$ de confianza para las variables de estrés general y laboral, depresión y ansiedad. Se contrastaron hipótesis mediante las pruebas $\mathrm{t}$ de Student para las variables de intervalo, y chi cuadrado y odds ratios para las variables categóricas. También se aplicó un modelo de regresión logística para evaluar el efecto de diferentes covariables en las variables fundamentales de la salud mental en relación al estrés general. En todos los casos se consideró un nivel de significancia máximo del $5 \%$. Los análisis fueron realizados con el paquete estadístico SPSS versión 15. 


\section{RESULTADOS}

\section{CARACTERÍSTICAS GENERALES DE LA POBLACIÓN}

De los 287 magistrados entrevistados, el $53 \%$ eran varones; el $25 \%$ era menor de 40 años y $50 \%$ entre 50 a más años. Con relación al estado civil, $68 \%$ era casado, $20 \%$, soltero y $18 \%$ correspondía a la categoría "otros" (viudos, divorciados o convivientes). En cuanto al periodo de antigüedad como magistrado, $34 \%$ tenía 10 o más años, $35 \%$ entre 5 y 9 años, y $31 \%$ menos de 5 años.

Con relación al cargo, en el Ministerio Público, 58,7 \% correspondía a fiscal provincial adjunto, $26,1 \%$ a fiscal provincial y $15,2 \%$ a fiscal superior, supremo y adjunto supremo. En el Poder Judicial, 18,8 \% a juez de paz letrado, $68,5 \%$ a juez especializado y $12,7 \%$ a vocal superior.

Durante la evaluación médica, se determinó que el 62,2 \% tenía sobrepeso, aunque esta condición era mayor en los magistrados del Poder Judicial $(66,7 \%)$ que en el Ministerio Público (56,9\%). Mientras, la prevalencia de presión arterial elevada fue de 20,2 \%; aunque difirió según la institución donde labora el Magistrado, siendo menor en el Ministerio Público (13\%) que en el Poder Judicial (26,8 \%).

\section{ESTRÉS GENERAL Y LABORAL}

La prevalencia de estrés general encontrada fue de 18,5 \% (IC95\%: 13,9 a 23,0 \%) en el nivel alto; aunque la prevalencia fue mayor en el Ministerio Público (19,6 \%) que en el Poder Judicial $(17,4 \%)$ y mayor en el sexo masculino (23\%) que en el femenino (14,5\%).

La prevalencia de estrés laboral se determinó en 33,7 $\%$; aunque esta fue mayor en varones $(43,9 \%)$, y en magistrados entre 5 a 10 años de servicio (43,3\%); las demás características se observan en la Tabla 1.

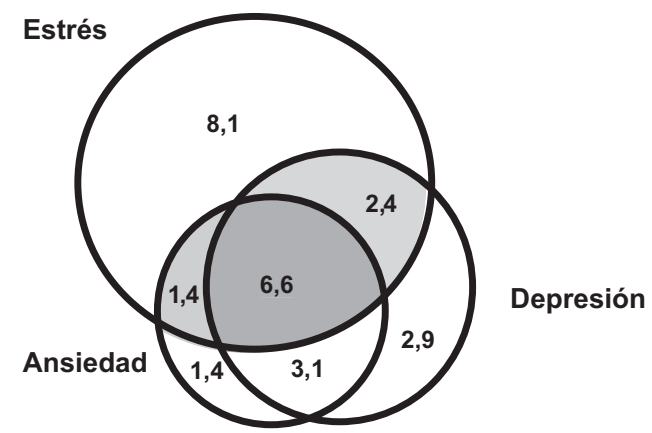

Figura 1. Porcentajes de estrés general, depresión y ansiedad en magistrados de Lima.

\section{ANSIEDAD Y DEPRESIÓN}

La prevalencia de ansiedad fue de 12,5\% (IC $95 \%$ : 8,7 a $16,4 \%)$; menor en el sexo masculino $(11,9 \%)$ que en el femenino $(13,2 \%)$.

La prevalencia de depresión fue de 15,0 \% (IC $95 \%$ : $10,8$ a $19,1 \%)$ : menor en el sexo masculino $(12,6 \%)$ que en el femenino $(17,1 \%)$.

Al calcular el riesgo en relación al nivel de estrés laboral, se identificó que un mayor nivel de estrés laboral está relacionado con un mayor nivel de depresión (OR: 4,87; IC $95 \%: 1,57-15,09$ ).

\section{ASOCIACIÓN DE VARIABLES}

La ansiedad, depresión y estrés general, estuvieron presentes en el $6,6 \%$ de los magistrados encuestados; mientras que el $25,9 \%$ tenían al menos una de estas condiciones.

Se aprecia una asociación entre el nivel de estrés general y los niveles de ansiedad $(p<0,001)$ y depresión $(p<0,001)$. En el análisis bivariado el estrés general se asoció con un riesgo 12,3 veces mayor de tener depresión (IC: 5,92-25,52), mientras la ansiedad lo estuvo en 13,0 veces (IC: $5,98-28,4)$. Al realizar el

Tabla 1. Niveles de estrés general, estrés laboral, depresión y ansiedad, según sexo, edad y años de magistrado

\begin{tabular}{lcccc}
\hline \multicolumn{1}{c}{ Características } & Estrés general (\%) & Estrés laboral (\%) & Depresión (\%) & Ansiedad (\%) \\
\hline TOTAL & 18,5 & 33,7 & 15,0 & 12,5 \\
Sexo & & & & \\
$\quad$ Masculino & 23,0 & 43,9 & 12,6 & 11,9 \\
$\quad$ Femenino & 14,5 & 25,5 & 17,1 & 13,2 \\
Edad & & & & \\
$\quad<40$ & 14,9 & 21,7 & 14,9 & 10,4 \\
40 a $<50$ & 20,6 & 42,5 & 12,5 & 11,8 \\
50 a más & 17,1 & 30,8 & 18,4 & 15,8 \\
Años de magistrado & & & & \\
$\quad 5$ & 20,0 & 30,8 & 16,5 & 10,6 \\
5 a $<10$ & 23,2 & 43,3 & 15,8 & 15,8 \\
10 a más & 11,0 & 25,8 & 13,2 & 12,1 \\
\hline
\end{tabular}


Tabla 2. Asociación entre estrés general y otras condiciones en magistrados de Lima, Perú.

\begin{tabular}{lccc}
\hline Variables & $\begin{array}{c}\text { OR crudo } \\
\text { (IC 95 \%) }\end{array}$ & $\begin{array}{c}\text { OR ajustado } \\
\text { (IC 95 \%) }\end{array}$ & $\boldsymbol{p}$ \\
\hline Depresión & $12,29(5,92-25,52)$ & $4,87(1,57-15,09)$ & 0,006 \\
Ansiedad & $13,03(5,98-28,42)$ & $8,48(2,52-28,60)$ & 0,001 \\
Sobrepeso & $0,72(0,37-1,41)$ & $0,40(0,16-1,01)$ & 0,052 \\
Sexo & $1,76(0,96-3,22)$ & $3,28(1,26-8,53)$ & 0,015 \\
\hline
\end{tabular}

análisis multivariado, ajustado con sobrepeso y sexo, el OR para depresión fue de 4,9 $(1,6-15,1)$ y de ansiedad de $8,5(2,5-28,6)$. Los valores ajustados para sobrepeso y sexo están en la Tabla 2.

\section{DISCUSIÓN}

En medicina, el estrés corresponde al estado de tensión física y psicológica que sufren los seres vivos en su proceso de adaptación al medio que los rodea. Los factores que lo generan se denominan "estresores", y el entorno en el cual aparecen, "situación estresante". El estrés ha sido estudiado bajo diferentes modelos explicativos. Hans Selye, propulsor del primero de ellos y creador del concepto que lo condujo a formular su teoría general de adaptación ${ }^{(15)}$, plantea en esta en tres fases: alarma, resistencia y agotamiento.

La teoría de Selye considera que todo ser vivo existe en continuo estrés pues la vida constituye un permanente esfuerzo de adaptación; es decir, vivir es adaptarse a las exigencias del ambiente utilizando los mecanismos fisiológicos que el organismo proporciona. A estos mecanismos fisiológicos deben añadirse los de orden psicológico, derivados de lo que, en general, denominamos salud mental. En 1974, Selye resumió su teoría, explicándola como una respuesta general inespecífica, que puede ser originada por eventos psicológicos y reducida por varias técnicas terapéuticas ${ }^{(16)}$.

Lazarus y Folkman (17) añadieron al modelo una interesante variable. Propusieron que la intensidad de esta depende del significado que el sujeto asigne al estresor, sin que necesariamente refleje con exactitud lo que en la realidad ocurre. Una persona puede interpretar una situación como amenazante (estrés) sin que en realidad sea así; es decir, el sujeto puede distorsionar lo que percibe. Desde esta perspectiva, los autores citados lo definen así: El estrés es una reacción particular entre el individuo y su entorno, evaluada por aquél como amenazante o desbordante de sus recursos poniendo en peligro su bienestar. Es decir, que el nivel de estrés aumentará en la medida que el sujeto considere que la situación supere sus posibilidades de control.

Finalmente, Valdés y De Flores ${ }^{(18)}$ señalan: el estrés es una activación autonómica y neuroendocrina, de naturaleza displacentera, que implica un fracaso adaptativo con cognición de indefensión e inhibición inmunológica y conductual. El saberse indefensos es la vivencia del sujeto de no poder hacer nada ante el peligroy debe rendirse ante él. La inhibición inmunológica reduce los mecanismos biológicos de defensa aumentado el riesgo a sufrir enfermedades, particularmente aquellas a las que se tenga una predisposición genética.

Desde el punto de vista clínico, cada persona tiene un umbral de percepción del estrés que depende de diversos factores, como son: su estado físico; su experiencia personal y condicionamientos previos; la calidad e intensidad de los estresores; su grado de distorsión de la situación estresante; la eficacia de sus mecanismos de respuesta (fisiológicos y psicológicos); su locus de control (es decir, si para el manejo del estrés el sujeto depende fundamentalmente de la ayuda de otros -locus externo- o si está convencido que puede manejarlo solo -locus interno-); y su soporte social (la ayuda real y el soporte afectivo disponibles).

Todo ello se resume en la siguiente fórmula: estrésvulnerabilidad-enfermedad, lo que significa que, si bien el estrés es parte de la vida normal, el exceso constituye una amenaza para la salud del sujeto, pues, de ceder sus resistencias, particularmente en sus puntos vulnerables (predisposiciones genéticas), se desencadenará la enfermedad en el momento que sus mecanismos de adaptación resulten insuficientes.

La literatura internacional destaca la importancia del estrés en enfermedades físicas, sospechando mecanismos inflamatorios ${ }^{(19)}$. En el campo de la salud mental, algunos autores lo consideran como paso previo a la enfermedad psiquiátrica y, otros, como un trastorno específico ${ }^{(1,20)}$.

El estrés laboral, que está presente en 33,7 \% de los magistrados, se explica por cuatro factores que podrían tener una explicación independiente: a) Los relacionados con el ambiente laboral, particularmente vinculados a la alta carga procesal con apoyo logístico insuficiente, que los obliga a un constante esfuerzo de alcance de metas presionados por límites de tiempo; b) La presión social ejercida por la opinión pública y los abogados litigantes, ante los cuales, en muchos casos, se sienten sin poder de acción; c) Las tensiones vinculadas con la superación profesional y la estabilidad en el cargo, pues la presión que sienten respecto al proceso de 
evaluación y ratificación del Consejo Nacional de la Magistratura es muy alta, donde no aprobar, implica cese en sus labores y un futuro incierto. La búsqueda de nueva ubicación laboral, problema serio en un país en desarrollo, especialmente si ya se tiene una carga familiar y un cómodo nivel de vida, exigirá nueva carga de estrés y esfuerzos de adaptación; d) El trato que reciben de parte de sus superiores, en el cual la queja de los magistrados se vincula con una percepción de poco reconocimiento institucional al mérito de sus esfuerzos y a una ausencia de mecanismos de soporte emocional en momentos difíciles.

Al respecto, Novaes y Sacramento, aunque con diferente metodología, encuentran en jueces laboralistas alta percepción de estrés, con un puntaje promedio de ocho en una escala decimal, y que los estresores más frecuentes se vinculan con sobrecarga de trabajo e interferencia con su vida familiar ${ }^{(4)}$. Mientras que Resnick, Myatt y Marotta encuentran que los estresores que afectan a los jueces se vinculan con el exceso de casos con severos límites de tiempo, aislamiento social, falta de privacidad y el peso emocional de tener que decidir el destino de otros ${ }^{(21)}$. $Y$ Tsai y Chan encuentran riesgo de burn out (síndrome de agotamiento) por estrés ocupacional en magistrados de Taiwan ${ }^{(22)}$, mientras que Rogers et al., en un estudio de 104 jueces canadienses encuestados anónimamente, así como 48 de sus esposas, vincularon los factores de estrés a establecer sentencias, asignar niños a custodia, tomar decisiones y al proceso del juicio especialmente con relación a los jurados ${ }^{(23)}$.

En el Perú, lamentablemente, no existe otra estimación de estrés laboral en magistrados con la cual pudiéramos comparar nuestros resultados. La única comparación posible sería con estrés laboral en población general adulta de Lima Metropolitana. Por ello, aunque con todas las limitaciones que impone el uso de diferentes metodologías y poblaciones de estudio, señalamos que el Estudio Epidemiológico de Salud Mental, realizado en Lima en el $2002{ }^{(24)}$, encontró entre los estresores vinculados "al trabajo o al estudio" (en otras palabras ocupacional) una prevalencia del $26 \%$. Insistimos en que, aunque los datos no son estrictamente comparables por las razones ya expuestas, el 33,7 \% de prevalencia de estrés laboral hallado en nuestro estudio aparece como un valor que requiere atención pues es mayor que en la población general.

Entre las condiciones médicas identificadas, si suponemos que todos los pacientes con presión arterial elevada en una sola toma tenían el diagnóstico de hipertensión arterial; la prevalencia de la hipertensión estaría dentro de los límites señalados por el Informe sobre Factores de Riesgo de las Enfermedades
Cardiovasculares en el Perú, 2006 (Estudio Tornasol) (25) para población general. Pero, llama la atención la alta prevalencia de sobrepeso (62,2 \%), en especial en el Poder Judicial (66,7\%).

De otro lado, la prevalencia de ansiedad encontrada fue de $12,5 \%$. El valor de referencia más aproximado para esta estimación es 7,3 \% correspondiente a población general de Lima, informada en la Encuesta de Salud Mental de Lima Metropolitana ${ }^{(24)}$. Nuevamente debemos señalar, como en el caso del estrés antes comentado, que aunque los datos no son estrictamente comparables por razones metodológicas, las diferencias apuntan a un hallazgo importante. Se destaca, además, que aquellos magistrados que practican actividades recreativas muestran menores niveles de ansiedad en comparación con los que no las practican, ello destaca el efecto protector contra la ansiedad $(p=0,001)$ de estas actividades.

Del mismo modo, el nivel de depresión en nuestro estudio fue de 15,0 \%. En el estudio metropolitano ya mencionado, encontraron una prevalencia de 6,6\% para episodios depresivos ${ }^{(24)}$. Reiteramos la limitación metodológica comparativa ya explicada. También respecto al nivel de depresión se observó el efecto protector de las actividades recreativas $(p=0,001)$, tal como actualmente se acepta en terapéutica psiquiátrica. Al respecto Neto Magalhães y França, encuentran una reducción de $21 \%$ en el nivel de estrés en estudiantes sometidos a un programa de ejercicios físicos de resistencia en comparación con el grupo control que no lo practicó ${ }^{(26)}$; por lo que las actividades físicas serían de gran ayuda terapéutica.

Por último, recordemos que en psiquiatría es frecuente observar el fenómeno de comorbilidad ${ }^{(27)}$. El hallazgo de la asociación del estrés general, ansiedad y depresión sugiere que estos tres factores se refuerzan $\mathrm{y}$, probablemente, incrementen así su potencial patogénico. Surge así, como hipótesis generada por nuestro estudio, que actuando estas variables en conjunto constituirían un factor de riesgo para la salud de los magistrados, particularmente de aquellos que por vulnerabilidad genética o psicológica, resulten más sensibles a su influencia. En tal perspectiva, un programa preventivo o correctivo, debería dirigirse a atender estas entidades y no solo a una de forma independiente, como suele ocurrir, por ejemplo, con los actuales programas antiestrés.

Deseamos señalar la necesidad de uniformar metodologías en estudios sobre estrés en magistrados, a fin de posibilitar estudios comparativos de resultados; la falta de investigación en este campo en el Perú 
es evidente, y nos limita para mejorar nuestras recomendaciones. El no disponer de escalas validadas para la evaluación de estrés o estrés laboral, limitan la precisión de los datos; en nuestro caso, empleamos una escala de Burnout diseñada para personas sometidas a trabajos muy exigentes, que podrían tolerar mejor el estrés; por ello las diferencias entre la prevalencia de estrés laboral y el estrés general en nuestro estudio. Por último, el diseño del estudio no permite afirmar con precisión las correlaciones mostradas, lo cual se muestra en los amplios intervalos de confianza mostrados.

En conclusión, en la población estudiada, el estrés laboral, ansiedad y depresión están presentes en una proporción moderada de magistrados; estando alguna de ellas presente en un cuarto de la población encuestada. Por tanto, se recomienda que los programas de intervención y prevención de trastornos en salud mental se dirijan al manejo del estrés, ansiedad y depresión en conjunto, dada su alta asociación.

\section{AGRADECIMIENTOS}

A los Drs. Maximiliano Cárdenas Díaz y Edmundo Peláez Bardales, expresidentes del Consejo Nacional de la Magistratura, por su constante apoyo y facilidades brindadas a la realización de este trabajo.

\section{Contribuciones de autoría}

APer: concepción del estudio, obtención del financiamiento, diseño, construcción de instrumento interpretación de resultados y redacción del trabajo. APad: construcción de instrumento, análisis e interpretación de datos y en la revisión del informe final. HC y LB: aplicación de encuestas, recolección de datos, revisión del informe final y obtención del financiamiento.

\section{Conflictos de interés}

Los autores declaran que haber tenido relación contractual con el Consejo Nacional de la Magistratura para la realización de la presente investigación.

\section{Fuentes de financiamiento}

Financiado por el Proyecto de Mejoramiento de los Servicios de Justicia, teniendo como institución participante al Consejo Nacional de la Magistratura (Contrato N. ${ }^{\circ}$ 026-2008/Cl).

\section{REFERENCIAS BIBLIOGRÁFÍCAS}

1. King M, Stanley G y Burrows G. Stress. Theory and Practice. Sydney: Grune and Stratton Inc.; 1987.
2. Perales A. Estudio de los niveles de estrés que afectan a la salud integral de los magistrados en la labor jurisdiccional y fiscal. Informe Final. Lima: Proyecto de Mejoramiento de los Servicios de Justicia; 2010

3. Brerner CF. Impact of a Mentoring Program on occupational stress, personal strain and coping resources of newly appointed U.S. Magistrate Judges [Tesis doctoral]. Des Moines, lowa, EEUU: Drake University, School of Education; 2002.

4. Novaes Lipp M, Sacramento Tanganelli M. Stress e Qualidade de Vida em Magistrados da Justiça do Trabalho: Diferenças entre Homens e Mulheres. Revista Psicologia Reflexão e Crítica. 2002;15(3):537-48.

5. Pereira J. Factores desencadeantes de estresse nos magistrados: o caso de Minas Gerais [Tesis de Maestría en Promoción de la Salud]. Sao Paulo, Brasil: Universidade de Franca; 2005.

6. Hunter TL. The relationship between judicial stress and job performance [Tesis doctoral]. California, EEUU: Alliant International University; 2007.

7. Salanova M. Trabajando con tecnologías y afrontando el tecnoestrés: el rol de las creencias de eficacia. Revista de Psicología del Trabajo y de las Organizaciones. 2003;19(3):225-46

8. Akiskal H. Mood Disorders: Introduction and Overview. En: Kaplan H, Sadock B (Eds). Textbook of Psychiatry. 6th edition. Vol. 1. Baltimore: Williams \& Wilkins; 1995.

9. Xu K, Ernst M, Goldman D. Imaging genomics applied to anxiety,stress response, and resiliency. Neuroinformatics. 2006;4(1):51-64.

10. Ehrenreich John H. The relief worker burnout questionnaire. Mental Health Workers without Borders; 2001.

11. Zung WWK. A self-rating depression scale. Arch Gen Psychiatry. 1965;(12):63-70.

12. Novara J, Sotillo C, Warthon D. Estandarización y correlación de las escalas de Beck, Hamilton y Zung para depresión en población de Lima Metropolitana. Lima: Instituto Nacional de Salud Mental; 1985.

13. Zung WWK. A rating Instrument for anxiety disorders. Psychosomatics. 1971;12(6):371-9.

14. Lusilla MP, Sánchez A, Sanz C, López J. Validación estructural de la escala heteroevaluada de ansiedad de Zung (XXVIII Congreso de la Sociedad Española de Psiquiatría). Anales de Psiquiatría. 1990;6(Sup 1):39.

15. Selye H. The Stress of life. New York: McGraw-Hill; 1956.

16. Selye H. Stress without distress. Philadelphia: J. B. Lippincott Co.; 1974.

17. Lazaruz RS, Folkman S. Estrés y procesos cognitivos. Barcelona: Ediciones Martínez Roca S.A; 1986.

18. Valdés M, De Flores T. Psicobiología del estrés. Barcelona: Ediciones Martínez Roca S.A.; 1990.

19. Black PH. The inflammatory consequences of psychologic stress: Relationship to insulin resistance, obesity, atherosclerosis and diabetes mellitus, type II. Med Hypotheses. 2006;67:879-91.

20. Rahe R. Stress and Psychiatry. En: Kaplan H, Sadock B. Comprehensive Textbook of Psychiatry. Baltimore: Williams \& Wilkins; 1995. 
21. Resnick A, Myatt K A, Marotta $P$ V. Surviving bench stress. Family Court Review. 2011;49(3):610-7.

22. Feng-Jen T, Chang-Chuan C. Occupational stress and burnout of judges and procurators. Int Arch Occup Environ Health. 2010;83(2):133-42.

23. Rogers JM, Freeman SJ, LeSage P. The occupational stress of judges. Can J Psychiatry. 1991;36(5):317-22.

24. Instituto Nacional de Salud Mental. EstudioEpidemiológico Metropolitano en Salud Mental, 2002. Informe General. Anales de Salud Mental. 2002;XVIII(1-2):1-197.

25. Segura L, Agusti R, Parodi J; Estudio TORNASOL. Factores de Riesgo de las Enfermedades Cardiovasculares en el Perú. Rev Peru Cardiol. 2006;XXXII(2):82-128.
26. Neto Magalhães AM, França NM. Efeitos de um programa de exercícios resistidos sobre o estresse mental em estudantes do Ensino Médio. R Bras Ci E Mov. 2003;11(4):33-6.

27. Popkin MK. Consultation-Liaison Psychiatry. En: Kaplan $\mathrm{H}$ \& Sadock B. Textbook of Psychiatry. Baltimore: Williams \& Wilkins; 1995.

\section{Correspondencia: Alberto Perales}

Dirección: Av. Javier Prado Oeste 445 Of. 101, Lima 27, Perú. Teléfono: (511) 4764939.

Correo electrónico: perales.alberto@gmail.com

\section{Suscríbase en forma electrónica y gratuita a los contenidos de la Revista Peruana de Medicina Experimental y Salud Pública, ingrese a WWW.ins.gob.pe, seleccione el ícono de la revista y envíenos sus datos.}

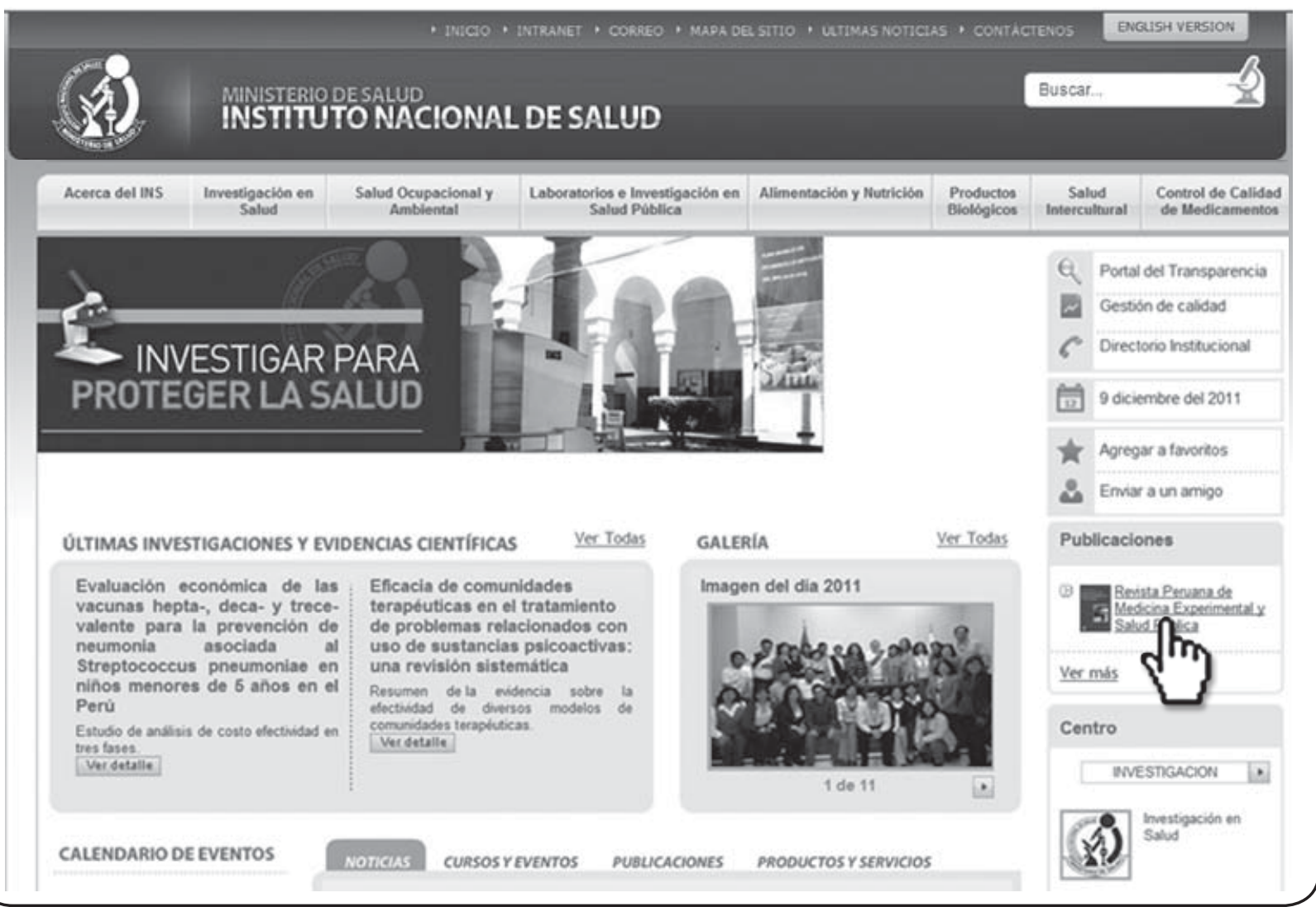

